\title{
Existence of Iterative Cauchy Fractional Differential Equation
}

\author{
Rabha W. Ibrahim \\ Institute of Mathematical Sciences, University Malaya, 50603 Kuala Lumpur, Malaysia \\ Correspondence should be addressed to Rabha W. Ibrahim; rabhaibrahim@yahoo.com
}

Received 15 December 2012; Revised 6 February 2013; Accepted 6 February 2013

Academic Editor: Jen-Chih Yao

Copyright (C) 2013 Rabha W. Ibrahim. This is an open access article distributed under the Creative Commons Attribution License, which permits unrestricted use, distribution, and reproduction in any medium, provided the original work is properly cited.

Our main aim in this paper is to use the technique of nonexpansive operators in more general iterative and noniterative fractional differential equations (Cauchy type). The noninteger case is taken in sense of the Riemann-Liouville fractional operators. Applications are illustrated.

\section{Introduction}

Fractional calculus and its applications (that is the theory of derivatives and integrals of any arbitrary real or complex order) are important in several widely diverse areas of mathematical, physical, and engineering sciences. It generalized the ideas of integer order differentiation and $n$-fold integration. Fractional derivatives introduce an excellent instrument for the description of general properties of various materials and processes. This is the main advantage of fractional derivatives in comparison with classical integer-order models, in which such effects are in fact neglected. The advantages of fractional derivatives become apparent in modeling mechanical and electrical properties of real materials, as well as in the description of properties of gases, liquids, rocks, and in many other fields.

Our aim in this paper is to consider the existence and uniqueness of nonlinear Cauchy problems of fractional order in sense of Riemann-Liouville operators. Also, two theorems in the analytic continuation of solutions are studied. In the fractional Cauchy problems, we replace the first-order time derivative by a fractional derivative. Fractional Cauchy problems are useful in physics. Recently, the author studied the the fractional Cauchy problems in complex domain [1].

One of the most frequently used tools in the theory of fractional calculus is furnished by the Riemann-Liouville operators. The Riemann-Liouville fractional derivative could hardly pose the physical interpretation of the initial conditions required for the initial value problems involving fractional differential equations. Moreover, this operator possesses advantages of fast convergence, higher stability, and higher accuracy to derive different types of numerical algorithms (see [2]).

Definition 1. The fractional (arbitrary) order integral of the function $f$ of order $\alpha>0$ is defined by

$$
I_{a}^{\alpha} f(t)=\int_{a}^{t} \frac{(t-\tau)^{\alpha-1}}{\Gamma(\alpha)} f(\tau) d \tau
$$

When $a=0$, we write $I_{a}^{\alpha} f(t)=f(t) * \phi_{\alpha}(t)$, where $(*)$ denoted the convolution product (see [3]), $\phi_{\alpha}(t)=$ $t^{\alpha-1} / \Gamma(\alpha), t>0$ and $\phi_{\alpha}(t)=0, t \leq 0$ and $\phi_{\alpha} \rightarrow \delta(t)$ as $\alpha \rightarrow 0$ where $\delta(t)$ is the delta function.

Definition 2. The fractional (arbitrary) order derivative of the function $f$ of order $0 \leq \alpha<1$ is defined by

$$
D_{a}^{\alpha} f(t)=\frac{d}{d t} \int_{a}^{t} \frac{(t-\tau)^{-\alpha}}{\Gamma(1-\alpha)} f(\tau) d \tau=\frac{d}{d t} I_{a}^{1-\alpha} f(t) .
$$

Remark 3. From Definitions 1 and 2, we have

$$
\begin{gathered}
D^{\alpha} t^{\mu}=\frac{\Gamma(\mu+1)}{\Gamma(\mu-\alpha+1)} t^{\mu-\alpha}, \quad \mu>-1,0<\alpha<1, \\
I^{\alpha} t^{\mu}=\frac{\Gamma(\mu+1)}{\Gamma(\mu+\alpha+1)} t^{\mu+\alpha}, \quad \mu>-1, \alpha>0 .
\end{gathered}
$$


Definition 4. The Caputo fractional derivative of order $\mu>0$ is defined, for a smooth function $f(t)$, by

$$
{ }^{c} D^{\mu} f(t):=\frac{1}{\Gamma(n-\mu)} \int_{0}^{t} \frac{f^{(n)}(\zeta)}{(t-\zeta)^{\mu-n+1}} d \zeta,
$$

where $n=[\mu]+1$, (the notation $[\mu]$ stands for the largest integer not greater than $\mu$ ).

Note that there is a relationship between Riemann-Liouville differential operator and the Caputo operator

$$
D_{a}^{\mu} f(t)=\frac{1}{\Gamma(1-\mu)} \frac{f(a)}{(t-a)^{\mu}}+{ }^{c} D_{a}^{\mu} f(t),
$$

and they are equivalent in a physical problem (i.e., a problem which specifies the initial conditions, that is, if $f(0)=0$, then the Riemann-Liouville derivative and the Caputo derivative of order $\alpha \in(0,1)$ coincide).

\section{Preliminaries}

We extract here the basic theory of nonexpansive mappings in order to offer the notions and results that will be needed in the next sections of the paper. Let $(X, d)$ be a metric space. A mapping $P: X \rightarrow X$ is said to be an $\nu$-contraction if there exists $\nu \in[0,1)$ such that

$$
d(P x, P y) \leq v d(x, y), \quad \forall x, y \in X .
$$

In the case where $v=1$, the mapping $P$ is said to be nonexpansive. Let $K$ be a nonempty subset of a real normed linear space $E$ and $P: K \rightarrow K$ be a map. In this setting, $P$ is nonexpansive if

$$
\|P x-P y\| \leq\|x-y\|, \quad \forall x, y \in K .
$$

The following result is a fixed point theorem for non expansive mappings, according to Berinde; see for example [4].

Theorem 5. Let $K$ be a nonempty closed convex and bounded subset of a uniformly Banach space $E$. Then any nonexpansive mapping $P: K \rightarrow K$ has at least a fixed point.

Definition 6. Let $K$ be a convex subset of a normed linear space $E$ and let $P: K \rightarrow K$ be a self-mapping. Given an $x_{0} \in K$ and a real number $\lambda \in[0,1]$, the sequence $x_{n}$ defined by the formula

$$
x_{n+1}=(1-\lambda) x_{n}+\lambda P x_{n}, \quad n=0,1,2, \ldots
$$

is usually called Krasnoselskij iteration or KrasnoselskijMann iteration.

Definition 7. Let $K$ be a convex subset of a normed linear space $E$ and let $P: K \rightarrow K$ be a self-mapping. Given an $x_{0} \in K$ and a real number $\lambda_{n} \in[0,1]$, the sequence $x_{n}$ defined by the formula

$$
x_{n+1}=\left(1-\lambda_{n}\right) x_{n}+\lambda_{n} P x_{n}, \quad n=0,1,2, \ldots
$$

is usually called Mann iteration.
Edelstein [5] proved that strict convexity of $E$ suffices for the Krasnoselskij iteration converge to a fixed point of $P$. While, Egri and Rus [6] proved that for any subset of $E$, the Mann iteration converge to a fixed point of $P$ when $P$ is a nonexpansive mapping.

We need the following results, which can be found in [7].

Lemma 8. Let $K$ be a convex and compact subset of a Banach space $E$ and let $P: K \rightarrow K$ be a non-expansive mapping. If the Mann iteration process $x_{n}$ satisfies the assumptions

(a) $x_{n} \in K$ for all positive integers $n$,

(b) $0 \leq \lambda_{n} \leq b<1$ for all positive integers $n$,

(c) $\sum_{n=0}^{\infty} \lambda_{n}=\infty$.

Then $x_{n}$ converges strongly to a fixed point of $P$.

Lemma 9. Let $K$ be a closed bounded convex subset of a real normed space $E$ and $P: K \rightarrow K$ a non-expansive mapping. If I - $P$ maps closed bounded subset of $E$ into closed subset of $E$ and $x_{n}$ is the Mann iteration, with $\lambda_{n}$ satisfying assumptions (a)-(c) in Lemma 8, then $x_{n}$ converges strongly to a fixed point of $P$ in $K$.

\section{Existence Theorems and Approximation of Solutions}

For most of the differential and integral equations with deviating arguments that appear in recent literature, the deviation of the argument usually involves only the time itself. However, another case, in which the deviating arguments depend on both the state variable $u$ and the time $t$, is of importance in theory and practice. Equations of the form

$$
u^{\prime}(t)=f(t, u(u(t)))
$$

are called iterative differential equations. These equations are important in the study of infection models and are related to the study of the motion of charged particles with retarded interaction (see $[3,8,9]$ ).

In this section, we establish the existence and uniqueness results for the fractional differential equation

$$
D^{\alpha}\left(u(t)-\mu t^{\alpha}\right)=f(t, u(u(t))),
$$

with initial condition $u(0)=0$, where $t, \mu \Gamma(\alpha+1):=u_{0} \in$ $J:=[0, T]$ and $f \in C(J \times J, J)$. For $t \in J$ denote

$$
\begin{gathered}
M_{t}=\max \{t, T-t\} \\
C_{L, \alpha}=\left\{u:\left|u\left(t_{1}\right)-u\left(t_{2}\right)\right|\right. \\
\left.\leq \frac{L}{\Gamma(\alpha+1)}\left|t_{1}-t_{2}\right|^{\alpha}, \forall t_{1}, t_{2} \in J\right\}, L>0 .
\end{gathered}
$$

It is clear that $C_{L, \alpha}$ is a nonempty convex and compact subset of the Banach space $(C[J],\|\cdot\|)$, where $\|x\|=\sup _{t \in J}|x(t)|$. 
Theorem 10. Assume that the following conditions are satisfied for the initial value problem (11):

(A1) $f \in C[J \times J, J]$;

(A2) $\exists \ell>0:|f(t, u)-f(t, v)| \leq \ell|u-v|$, for all $t, u, v \in J$;

(A3) if $L$ is the Lipschitz constant such that $\left|u\left(t_{1}\right)-u\left(t_{2}\right)\right| \leq$ $(L / \Gamma(\alpha+1))\left|t_{1}-t_{2}\right|^{\alpha}$, then

$$
M=\max \{|f(t, u)|:(t, u) \in J \times J\} \leq \frac{L}{2} ;
$$

(A4) one of the following conditions holds:

(a) $M\left(T^{\alpha} / \Gamma(\alpha+1)\right) \leq M_{u_{0}}$, where $M_{u_{0}}=\max \left\{u_{0}\right.$, $\left.T-u_{0}\right\}$

(b) $u_{0}=0, M\left(T^{\alpha} / \Gamma(\alpha+1)\right) \leq T-u_{0}, f(t, u) \geq 0$, for all $t, u \in J$;

(c) $u_{0}=T, M\left(T^{\alpha} / \Gamma(\alpha+1)\right) \leq u_{0}, f(t, u) \geq 0$, for all $t, u \in J$.

If

$$
\frac{(\widetilde{L}+1) T^{\alpha} \ell}{\Gamma(\alpha+1)} \leq 1,
$$

then there exists at least one solution of problem (11) in $C_{L, \alpha}$ which can be approximated by the Krasnoselskij iteration

$$
u_{n+1}=(1-\lambda) u_{n}+\lambda u_{0}+\lambda \int_{0}^{t} \frac{(t-\tau)^{\alpha-1}}{\Gamma(\alpha)} f(\tau, u(u(\tau))) d \tau
$$

where $\lambda \in(0,1)$ and $u_{1} \in C_{L, \alpha}$ are arbitrary.

Proof. Consider the integral operator $P: C_{L, \alpha} \rightarrow C(J)$

$$
\begin{array}{r}
P u(t)=u_{0}+\int_{0}^{t} \frac{(t-\tau)^{\alpha-1}}{\Gamma(\alpha)} f(\tau, u(u(\lambda \tau))) d \tau, \\
t \in J, u \in C_{L, \alpha} .
\end{array}
$$

Our aim is to show that $P$ has a fixed point in $C_{L, \alpha}$. We proceed to apply Schauder fixed point theorem or Banach fixed point theorem.

First we show that $C_{L, \alpha}$ is invariant set with respect to $P$, that is, $T\left(C_{L, \alpha}\right) \subset C_{L, \alpha}$. In virtue of condition (A4(a)) and for all $t \in J, u \in C_{L, \alpha}$, we have

$$
\begin{aligned}
& |P u(t)| \\
& \quad \leq u_{0}+\left|\int_{0}^{t} \frac{(t-\tau)^{\alpha-1}}{\Gamma(\alpha)} f(\tau, u(u(\lambda \tau))) d \tau\right| \\
& \quad \leq u_{0}+M \frac{T^{\alpha}}{\Gamma(\alpha+1)} \leq T,
\end{aligned}
$$

$|P u(t)|$

$$
\begin{aligned}
& \geq u_{0}-\left|\int_{0}^{t} \frac{(t-\tau)^{\alpha-1}}{\Gamma(\alpha)} f(\tau, u(u(\lambda \tau))) d \tau\right| \\
& \geq u_{0}-M \frac{T^{\alpha}}{\Gamma(\alpha+1)} \geq u_{0}-M_{u_{0}} \geq 0 .
\end{aligned}
$$

Thus $(P u)(t) \in J, t \in J$. In the similar manner of (A4(a)), we treat the cases (A4(b)) and (A4(c)). Now for every $t_{1}, t_{2} \in$ $J$, by (A3), we obtain

$$
\begin{aligned}
\mid(P u) & \left(t_{1}\right)-(P u)\left(t_{2}\right) \mid \\
= & \mid \int_{0}^{t_{1}} \frac{(t-\tau)^{\alpha-1}}{\Gamma(\alpha)} f(\tau, u(u(\lambda \tau))) d \tau \\
& \quad-\int_{0}^{t_{2}} \frac{(t-\tau)^{\alpha-1}}{\Gamma(\alpha)} f(\tau, u(u(\lambda \tau))) d \tau \mid \\
\leq & M \frac{\left|t_{1}^{\alpha}-t_{2}^{\alpha}+2\left(t_{1}-t_{2}\right)^{\alpha}\right|}{\Gamma(\alpha+1)} \\
\leq & 2 M \frac{\left|t_{1}-t_{2}\right|^{\alpha}}{\Gamma(\alpha+1)} \\
\leq & L \frac{\left|t_{1}-t_{2}\right|^{\alpha}}{\Gamma(\alpha+1)} .
\end{aligned}
$$

Hence $(P u) \in C_{L, \alpha}$ whenever $u \in C_{L, \alpha}$. Therefore, $P$ : $C_{L, \alpha} \rightarrow C_{L, \alpha}$ (i.e., $P$ is a self-mapping of $C_{L, \alpha}$ ). Let $u, v \in C_{L, \alpha}$ and $t \in J$, by employing (A2), we have

$$
\begin{aligned}
& |(P u)(t)-(P v)(t)| \\
& =\mid \int_{0}^{t} \frac{(t-\tau)^{\alpha-1}}{\Gamma(\alpha)} f(\tau, u(u(\lambda \tau))) d \tau \\
& \quad-\int_{0}^{t} \frac{(t-\tau)^{\alpha-1}}{\Gamma(\alpha)} f(\tau, v(v(\lambda \tau))) d \tau \mid \\
& \leq \int_{0}^{t} \frac{(t-\tau)^{\alpha-1}}{\Gamma(\alpha)} \mid f(\tau, u(u(\lambda \tau))) \\
& \leq \ell \int_{0}^{t} \frac{(t-\tau)^{\alpha-1}}{\Gamma(\alpha)}|u(u(\lambda \tau))-v(v(\lambda \tau))| d \tau \\
& =\ell \int_{0}^{t} \frac{(t-\tau)^{\alpha-1}}{\Gamma(\alpha)} \mid u(u(\lambda \tau))-u(v(\lambda \tau)) \\
& \leq \frac{T^{\alpha} \ell}{\Gamma(\alpha+1)}[\widetilde{L}+1]\|u-v\|, \\
& +(\lambda \tau(\nu))) \mid d \tau)-v(v(\lambda \tau)) \mid d \tau
\end{aligned}
$$

where

$$
\widetilde{L}=\max \frac{L}{\Gamma(\alpha+1)} .
$$

Now, by taking the supremum in the last assertion, we get

$$
\|(P u)-(P v)\| \leq \frac{(\widetilde{L}+1) T^{\alpha} \ell}{\Gamma(\alpha+1)}\|u-v\|
$$


If $(\widetilde{L}+1) T^{\alpha} \ell / \Gamma(\alpha+1)<1$, then $P$ is a contraction mapping and hence in view of Banach fixed point theorem, (11) has a unique solution. Now if

$$
\frac{(\widetilde{L}+1) T^{\alpha} \ell}{\Gamma(\alpha+1)}=1,
$$

then $P$ is non-expansive and, hence, continuous; thus Schauder fixed point theorem implies that (11) has a solution in $C_{L, \alpha}$. Finally, in view of Lemmas 8 and 9 , we obtain the second part of the theorem.

Next we establish the solution of (11) in a subset of $C_{L, \alpha}$ defined by

$$
\begin{array}{r}
C_{L, \alpha, \delta}=\left\{u \in C_{L, \alpha}: u(t) \leq \frac{\delta t^{\alpha}}{\Gamma(\alpha+1)}, \quad \forall t \in J\right\}, \\
\delta \in(0,1) .
\end{array}
$$

It is clear that $C_{L, \alpha, \delta}$ is nonempty, convex, and compact subset in $C[J]$.

Theorem 11. Assume that the following conditions are satisfied.

(A5) $u_{0} \leq\left(\delta t_{0}^{\alpha} / 2 \Gamma(\alpha+1)\right), t_{0}(\neq 0) \in J$.

(A6) If $L$ is the Lipschitz constant such that $\left|u\left(t_{1}\right)-u\left(t_{2}\right)\right| \leq$ $(L / \Gamma(\alpha+1))\left|t_{1}-t_{2}\right|^{\alpha}$, then $M \leq \min \{(\delta / 2),(L / 2)\}$.

(A7) There exists a $\bar{\tau}>0$ such that $\bar{\tau}>-(\ln (1-\delta) / \delta(T-$ $\left.\left.t_{0}\right)\right), T \neq t_{0}$ and

$$
\frac{T^{\alpha-1} \ell}{\Gamma(\alpha) \bar{\tau}}\left(\frac{1}{\delta}+\widetilde{L}\right) \max \left\{e^{\bar{\tau} t_{0}}-1,1-e^{\bar{\tau}\left(t_{0}-T\right)}\right\} \leq 1 .
$$

If (A2), (A4) hold then there exists at least one solution of problem (11) in $C_{L, \alpha, \delta}$ which can be approximated by the Krasnoselskij iteration

$$
u_{n+1}=(1-\lambda) u_{n}+\lambda u_{0}+\lambda \int_{0}^{t} \frac{(t-\tau)^{\alpha-1}}{\Gamma(\alpha)} f(\tau, u(u(\tau))) d \tau,
$$

where $\lambda \in(0,1)$ and $u_{1} \in C_{L, \alpha, \delta}$ are arbitrary.

Proof. We assume the Banach space $C[J]$ endowed with Bielecki's norm is given by the formula

$$
\begin{gathered}
\|u\|_{B}=\max _{t \in J}|u(t)| e^{-s\left(t-t_{0}\right)}, \quad s>0, t>t_{0} \\
\left(t, s, t_{0} \in J=[0, T], T<\infty\right) .
\end{gathered}
$$

Let $P$ be defined as in the proof of Theorem 10. By assumptions (A2), (A4), and (A6), it follows that

$$
P\left(C_{L, \alpha, \delta}\right) \subset C_{L, \alpha, \delta} .
$$

Now we prove that $C_{L, \alpha, \delta}$ is an invariant set with respect to the operator $P$. Indeed, if $u \in C_{L, \alpha, \delta}$ and $t \in J$ then in view of (A5) and (A6), we have

$$
\begin{aligned}
P u(t) & \leq u_{0}+M \frac{t^{\alpha}}{\Gamma(\alpha+1)} \\
& =u_{0}+M \frac{\left(t^{\alpha}-t_{0}^{\alpha}\right)+t_{0}^{\alpha}}{\Gamma(\alpha+1)} \\
& \leq \frac{\delta t_{0}^{\alpha}}{2 \Gamma(\alpha+1)}+\frac{\delta t^{\alpha}}{2 \Gamma(\alpha+1)}-\frac{\delta t_{0}^{\alpha}}{2 \Gamma(\alpha+1)}+\frac{\delta t_{0}^{\alpha}}{2 \Gamma(\alpha+1)} \\
& \leq \frac{\delta t^{\alpha}}{\Gamma(\alpha+1)}, \quad t>t_{0},
\end{aligned}
$$

that is, $P u \in C_{L, \alpha, \delta}$.

Let $u, v \in C_{L, \alpha, \delta}$ and $t \in J$, we have

$$
\begin{aligned}
& |(P u)(t)-(P v)(t)| \\
& =\mid \int_{0}^{t} \frac{(t-\tau)^{\alpha-1}}{\Gamma(\alpha)} f(\tau, u(u(\lambda \tau))) d \tau \\
& \quad-\int_{0}^{t} \frac{(t-\tau)^{\alpha-1}}{\Gamma(\alpha)} f(\tau, v(v(\lambda \tau))) d \tau \mid \\
& \leq \frac{T^{\alpha-1} \ell}{\Gamma(\alpha)} \mid \int_{0}^{t}(\widetilde{L}|u(\tau)-v(\tau)| \\
& \quad+|u(v(\lambda \tau))-v(v(\lambda \tau))|) d \tau \mid \\
& \leq \frac{T^{\alpha-1} \ell}{\Gamma(\alpha)}\left(\left|\int_{0}^{t} \widetilde{L} e^{s\left(\tau-t_{0}\right)} d \tau\right|\right. \\
& \left.\quad+\left|\int_{0}^{t} e^{s\left(\delta \tau-t_{0}\right)} d \tau\right|\right)\|u-v\|_{B} \\
& \leq \frac{T^{\alpha-1} \ell}{\Gamma(\alpha)}\left(\left|\widetilde{L}\left(e^{s\left(t-t_{0}\right)}-1\right)\right|\right. \\
& \left.\quad+\frac{1}{\delta s}\left|e^{s\left(\delta t-t_{0}\right)}-e^{s\left(\delta t_{0}-t_{0}\right)}\right|\right)\|u-v\|_{B} .
\end{aligned}
$$

This yields

$$
\begin{aligned}
& |(P u)(t)-(P v)(t)| e^{-s\left(\tau-t_{0}\right)} \\
& \leq \frac{T^{\alpha-1} \ell}{s \Gamma(\alpha)}\left(\widetilde{L}\left|1-e^{-s\left(t-t_{0}\right)}\right|\right. \\
& \left.\quad+\frac{1}{\delta}\left|e^{s(\delta-1) t}-e^{s\left(\delta t_{0}-t\right)}\right|\right)\|u-v\|_{B} \\
& \quad:=L(t)\|u-v\|_{B},
\end{aligned}
$$

where $L(t)$ is a continuous function. Then there exists a constant $\widehat{L}>0$ such that

$$
\max _{t \in J}|L(t)| \leq \widehat{L}
$$


Thus we have

$$
\|P u-P v\|_{B} \leq \widehat{L}\|u-v\|_{B},
$$

which shows that $P$ is Lipschitzian, hence continuous. By Schauder's fixed point theorem, it follows that $T$ has at least one fixed point which is actually a solution of the initial value problem (11).

We proceed to show that $P$ is nonexpansive function. The function

$$
g(t)=1-e^{-s\left(t-t_{0}\right)}, \quad s>0, t>t_{0}
$$

is strictly increasing on $J$ and $g\left(t_{0}\right)=0$; furthermore,

$$
\max _{t \in J} g(t)=\max \left\{e^{\tilde{\tau} t_{0}}-1,1-e^{\tilde{\tau}\left(t_{0}-T\right)}\right\} .
$$

Similarly for the function

$$
h(t)=e^{s(\delta-1) t}-e^{s\left(\delta t_{0}-t\right)}
$$

then

$$
h^{\prime}(t)=s e^{s(\delta-1) t}\left[(\delta-1)+e^{s \delta\left(t-t_{0}\right)}\right] .
$$

Now the function

$$
k(t)=(\delta-1)+e^{s \delta\left(t-t_{0}\right)}
$$

is strictly decreasing on $J$; hence,

$$
k(t) \geq k(T)=(\delta-1)+e^{s \delta\left(T-t_{0}\right)} .
$$

For $\delta \in(0,1)$ and $T \neq t_{0}$ then by the assumption (A7), there exists a $\bar{\tau}>0$ such that

$$
\bar{\tau}>-\frac{\ln (1-\delta)}{\delta\left(T-t_{0}\right)}, \quad T \neq t_{0}
$$

which implies that $k(T)>0$ and hence $h$ is strictly increasing on $J$. If we put $s=\bar{\tau}$, we have

$$
\max _{t \in J}|h(t)|=\max \left\{\left|1-e^{s \delta t_{0}}\right|,\left|e^{s(\delta-1) T}-e^{s\left(\delta t_{0}-T\right)}\right|\right\} .
$$

But since $\delta \in(0,1)$ thus we get

$$
\begin{aligned}
\left|e^{s(\delta-1) T}-e^{s\left(\delta t_{0}-T\right)}\right| & =e^{s(\delta-1) T}\left|1-e^{s \delta\left(t_{0}-T\right)}\right| \\
& \leq 1-e^{s \delta\left(t_{0}-T\right)},
\end{aligned}
$$

for sufficient $s, \delta, T$, and $t_{0}$. Moreover, we have

$$
\left|1-e^{s \delta t_{0}}\right| \leq e^{s \delta t_{0}}-1 .
$$

Consequently, we receive

$$
L(t) \leq \max \left\{e^{s \delta t_{0}}-1,1-e^{s \delta\left(t_{0}-T\right)}\right\} \frac{T^{\alpha-1} \ell}{s \Gamma(\alpha)}\left(\frac{1}{\delta}+\widetilde{L}\right) .
$$

This shows that $P$ is non-expansive.

Similar argument holds when $T=t_{0}$, in (38) we have $k(T)=\delta>0$, hence $h$ is strictly increasing on $J$. Finally, one can use Lemmas 8 and 9 to obtain the second part of the theorem. This completes the proof.
Example 12. Consider the following initial value problem associated to an fractional iterative differential equation

$$
\begin{gathered}
D^{0.5} u(t)=-\frac{1}{3}+\frac{1}{4} u(u(t)), \quad t \in[0,1] \\
u(0)=\frac{1}{3}
\end{gathered}
$$

where $u \in C^{1}([0,1],[0,1])$. We are focused on the solutions $u \in C^{1}([0,1],[0,1])$ belonging to the set

$$
\begin{aligned}
& C_{1,0.5}=\{u:\left|u\left(t_{1}\right)-u\left(t_{2}\right)\right| \\
& \leq\left.\frac{1}{\Gamma(3 / 2)}\left|t_{1}-t_{2}\right|^{0.5}, \forall t_{1}, t_{2} \in[0,1]\right\} \\
&=\left\{u:\left|u\left(t_{1}\right)-u\left(t_{2}\right)\right|\right. \\
& \leq\left.\frac{1}{0.886} \sqrt{\left|t_{1}-t_{2}\right|}, \forall t_{1}, t_{2} \in[0,1]\right\} \\
&=\left\{u:\left|u\left(t_{1}\right)-u\left(t_{2}\right)\right|\right. \\
&\left.\leq 1.1 \sqrt{\left|t_{1}-t_{2}\right|}, \forall t_{1}, t_{2} \in[0,1]\right\} .
\end{aligned}
$$

To satisfy (A4(a)), we have $M \leq L / 2 \simeq 1 / 2, M_{1 / 3}=$ $\max \{1 / 3,2 / 3\}=2 / 3=0.666$ and

$$
M \frac{T^{\alpha}}{\Gamma(\alpha+1)}=\frac{1}{2} \times \frac{1}{0.886}=0.56<0.666 .
$$

Hence (A4(a)) is satisfied. The function $f(t)=-(1 / 3)+$ $(1 / 4) u$ is Lipschitzian with the Lipschitz constant $\ell=1 / 4$. This shows that

$$
\frac{(\widetilde{L}+1) T^{\alpha} \ell}{\Gamma(\alpha+1)}=\frac{2.1 \times 0.25}{0.886}=0.592<1 .
$$

Therefore, by Theorem 10, we obtain information on the existence and approximation for the solutions of the initial value problem (44).

If we consider the function $f(t)=-(1 / 3)+(422 / 1000) u$ in Example 12, then we obtain

$$
\frac{(\widetilde{L}+1) T^{\alpha} \ell}{\Gamma(\alpha+1)}=\frac{2.1 \times 0.422}{0.886} \simeq 1 .
$$

Therefore, again by Theorem 10 we pose the existence and approximation of the solutions of the initial value problem (44).

Again, we consider the problem (44) on the interval $[(3 / 4), 1]$ for $\ell=0.025$, where $u \in C^{1}([(3 / 4), 1],[(3 / 4), 1])$. 
We are interested in the solutions $u \in C^{1}([3 / 4,1],[3 / 4,1])$ belonging to the set

$$
\begin{aligned}
& C_{1,(1 / 2),(3 / 4)} \\
& =\left\{u \in C_{1,(1 / 2)}: u(t)\right. \\
& \left.\quad \leq \frac{\delta t^{\alpha}}{\Gamma(\alpha+1)}, \forall t \in J\right\}, \quad \delta \in(0,1) \\
& =\left\{u: u(t) \leq \frac{(3 / 4) t^{(1 / 2)}}{\Gamma(3 / 2)}, \forall t \in[(3 / 4), 1]\right\} \\
& =\left\{u: u(t) \leq 0.846 \sqrt{t}, t \in\left[\frac{3}{4}, 1\right]\right\} .
\end{aligned}
$$

Our aim is to satisfy the assumptions of Theorem 11. (A2) and (A4) are valid. Since $u_{0}=1 / 3$ and $t_{0}=3 / 4$, we have

$$
u_{0} \leq \frac{\delta t_{0}^{\alpha}}{2 \Gamma(\alpha+1)} \Longrightarrow \frac{1}{3}<\frac{3}{8} ;
$$

hence (A5) is satisfied. Moreover, a computation gives

$$
M \leq \min \left\{\frac{\delta}{2}, \frac{L}{2}\right\}=\min \left\{\frac{\delta}{2}, \frac{L}{2}\right\}=\left\{\frac{3}{8}, \frac{1}{2}\right\}=\frac{3}{8}
$$

thus (A6) is satisfied. Now we proceed to satisfy (A7), since

$$
\begin{gathered}
-\frac{\ln (1-\delta)}{\delta\left(T-t_{0}\right)}=\frac{-\ln (1 / 4) \times 16}{3}=6.933, \\
\max \left\{e^{\bar{\tau} t_{0}}-1,1-e^{\bar{\tau}\left(t_{0}-T\right)}\right\}=\max \{189.5, .826\}
\end{gathered}
$$

then for $\bar{\tau}=7$, we impose

$$
\begin{aligned}
& \frac{T^{\alpha-1} \ell}{\Gamma(\alpha) \bar{\tau}}\left(\frac{1}{\delta}+\widetilde{L}\right) \max \left\{e^{\bar{\tau} t_{0}}-1,1-e^{\bar{\tau}\left(t_{0}-T\right)}\right\} \\
& \quad=\frac{0.175}{37.17} \max \{189.5, .826\} \\
& \quad=0.758<1 .
\end{aligned}
$$

Hence in view of Theorem 11, problem (44) has a solution in the set $C_{1,(1 / 2),(3 / 4)}$.

We can observe that problem (44) has not a solution on the set $C_{1,(1 / 2),(1 / 2)}$ over the interval [(1/2), 1]:

$$
\begin{aligned}
& C_{1,(1 / 2),(1 / 2)} \\
& =\left\{u \in C_{1,(1 / 2)}: u(t) \leq \frac{\delta t^{\alpha}}{\Gamma(\alpha+1)}, \quad \forall t \in J\right\}, \\
& \delta \in(0,1) \\
& =\left\{u: u(t) \leq \frac{(1 / 2) t^{(1 / 2)}}{\Gamma(3 / 2)}, \forall t \in\left[\frac{1}{2}, 1\right]\right\} \\
& =\left\{u: u(t) \leq 0.564 \sqrt{t}, t \in\left[\frac{1}{2}, 1\right]\right\} .
\end{aligned}
$$

For $u_{0}=(1 / 3), t_{0}=(1 / 2), \alpha=(1 / 2), \delta=(1 / 2)$, a calculation poses

$$
u_{0} \leq \frac{\delta t_{0}^{\alpha}}{2 \Gamma(\alpha+1)} \Longrightarrow \frac{1}{3}>\frac{0.35}{1.772} ;
$$

therefore, condition (A5) dose not satisfy.

Finally, problem (44) has not a solution on the set $C_{1,(1 / 2),(1 / 2)}$ over the interval $[(3 / 4), 1]$ : (in view of Theorem 11)

$$
\begin{aligned}
& C_{1,(1 / 2),(1 / 2)} \\
& =\left\{u \in C_{1,(1 / 2)}: u(t) \leq \frac{\delta t^{\alpha}}{\Gamma(\alpha+1)}, \forall t \in J\right\}, \\
& \delta \in(0,1) \\
& =\left\{u: u(t) \leq \frac{(1 / 2) t^{(1 / 2)}}{\Gamma(3 / 2)}, \forall t \in\left[\frac{3}{4}, 1\right]\right\} \\
& =\left\{u: u(t) \leq 0.5 \sqrt{t}, t \in\left[\frac{3}{4}, 1\right]\right\} .
\end{aligned}
$$

For $u_{0}=(1 / 3), t_{0}=(3 / 4), \alpha=(1 / 2), \delta=(1 / 2)$, a calculation yields

$$
u_{0} \leq \frac{\delta t_{0}^{\alpha}}{2 \Gamma(\alpha+1)} \Longrightarrow \frac{1}{3}>\frac{1}{4}
$$

therefore, condition (A5) dose not satisfy.

As such iterative fractional differential equations are used to generalize the model infective disease processes, pattern formation in the plane, and are important in investigations of dynamical systems, future works will be also devoted to them.

\section{Acknowledgment}

The author is thankful to the anonymous referee for his/her helpful suggestions for the improvement of this paper.

\section{References}

[1] R. W. Ibrahim, "Existence and uniqueness of holomorphic solutions for fractional Cauchy problem," Journal of Mathematical Analysis and Applications, vol. 380, no. 1, pp. 232-240, 2011.

[2] A. A. Kilbas, H. M. Srivastava, and J. J. Trujillo, Theory and applications of Fractional Differential Equations, vol. 204 of North-Holland Mathematics Studies, Elsevier, 2006.

[3] D. Yang and W. Zhang, "Solutions of equivariance for iterative differential equations," Applied Mathematics Letters, vol. 17, no. 7, pp. 759-765, 2004.

[4] V. Berinde, Iterative Approximation of Fixed Points, vol. 1912, Springer, Berlin, Germany, 2nd edition, 2007.

[5] M. Edelstein, "A remark on a theorem of M. A. Krasnoselski," The American Mathematical Monthly, vol. 73, pp. 509-510, 1966.

[6] E. Egri and I. A. Rus, "First order iterative functional-differential equation with parameter," Studia Universitatis Babeş-Bolyai Mathematica, vol. 52, no. 4, pp. 67-80, 2007.

[7] C. Chidume, Geometric Properties of Banach Spaces and Nonlinear Iterations, vol. 1965, Springer, Berlin, Germany, 2009. 
[8] A. Rontó and M. Rontó, "Successive approximation method for some linear boundary value problems for differential equations with a special type of argument deviation," Miskolc Mathematical Notes, vol. 10, no. 1, pp. 69-95, 2009.

[9] V. Berinde, "Existence and approximation of solutions of some first order iterative differential equations," Miskolc Mathematical Notes, vol. 11, no. 1, pp. 13-26, 2010. 


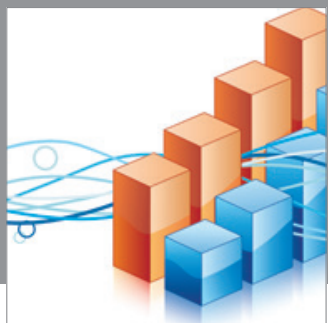

Advances in

Operations Research

mansans

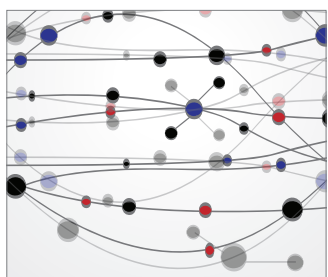

The Scientific World Journal
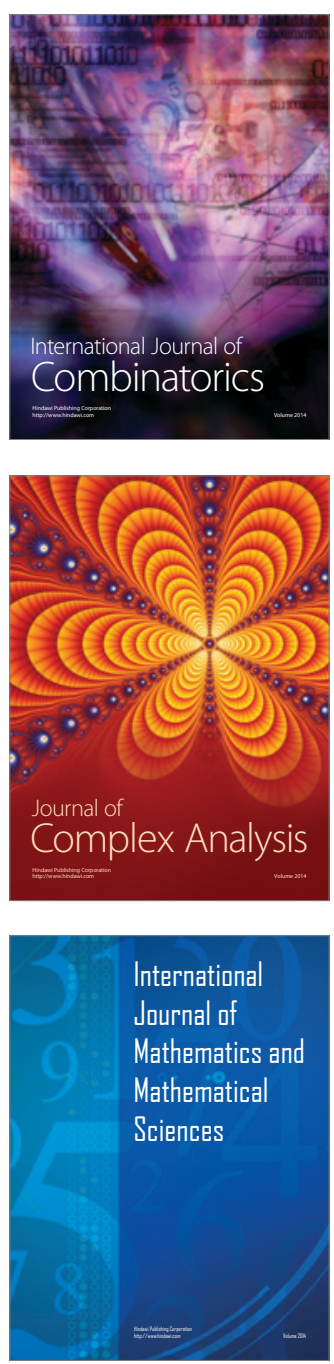
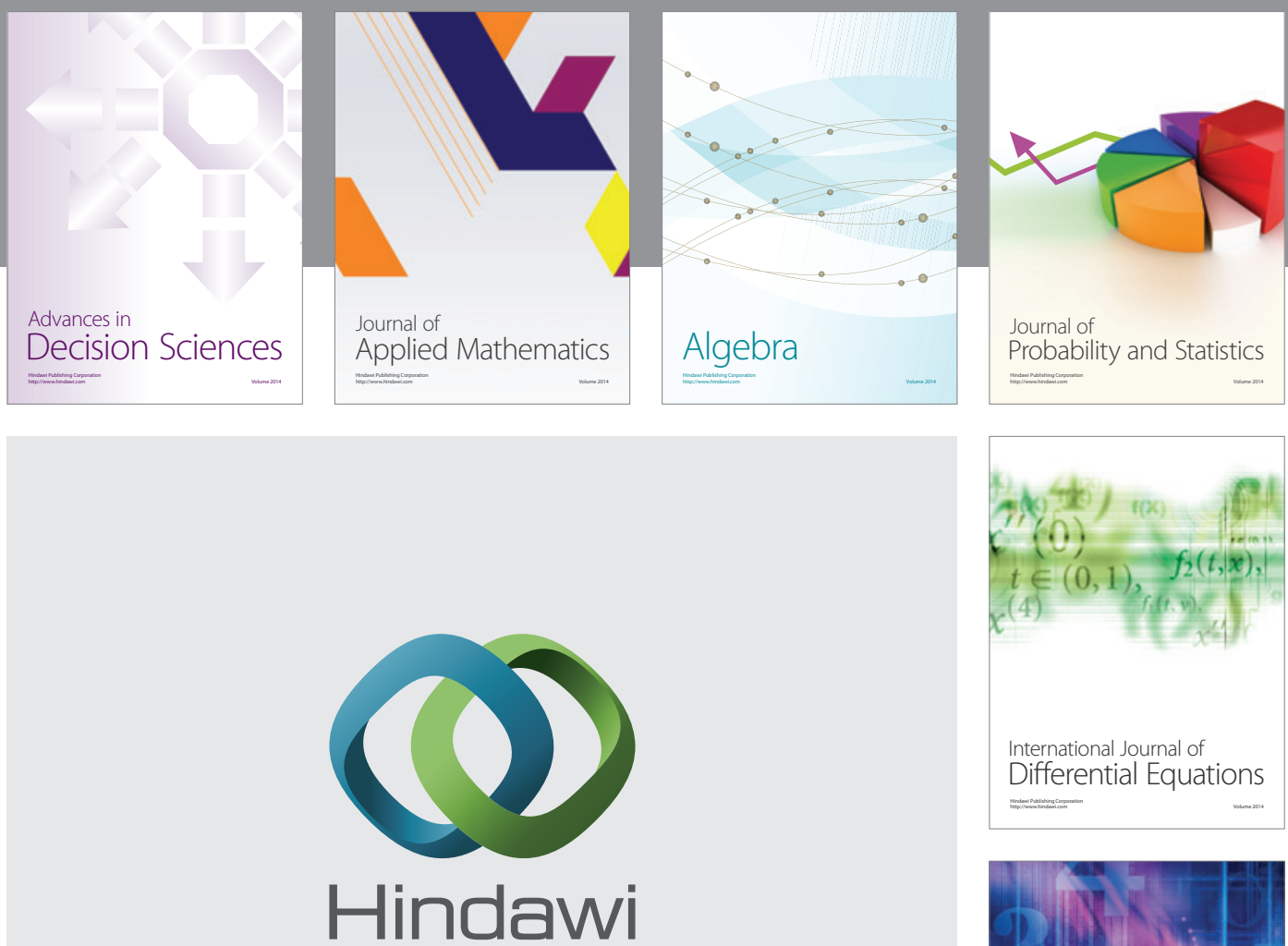

Submit your manuscripts at http://www.hindawi.com
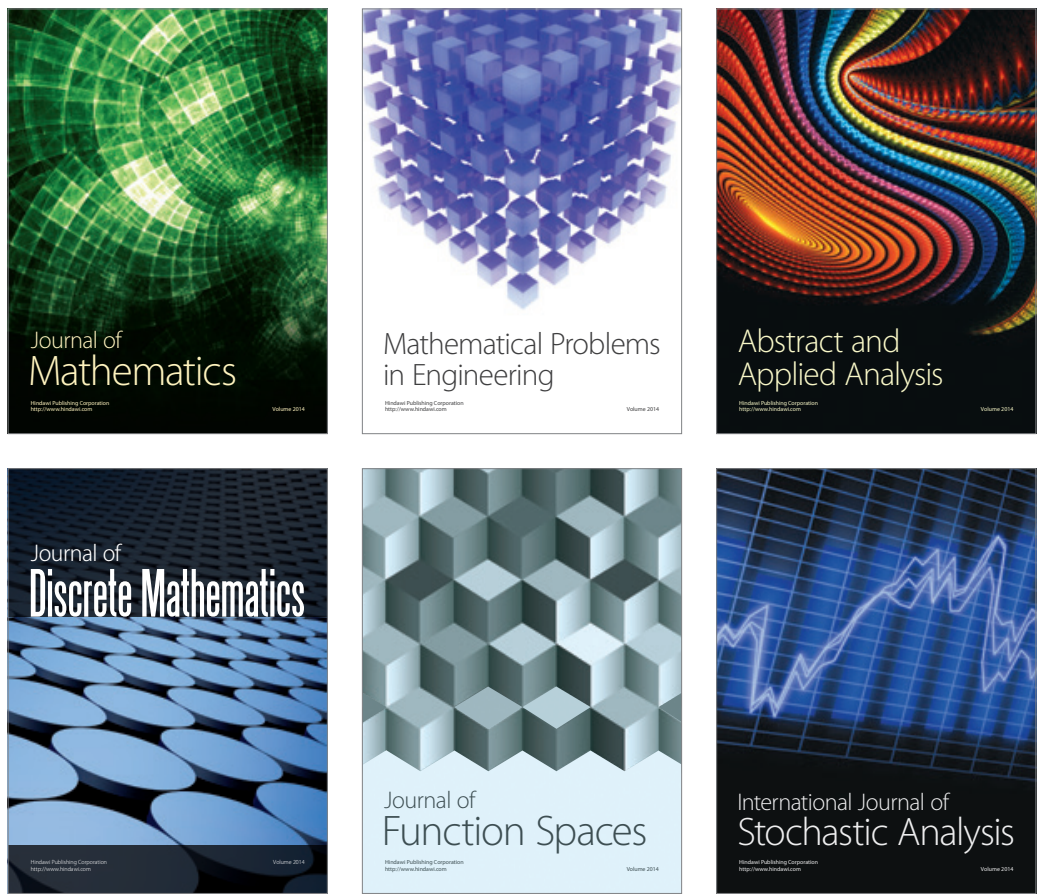

Journal of

Function Spaces

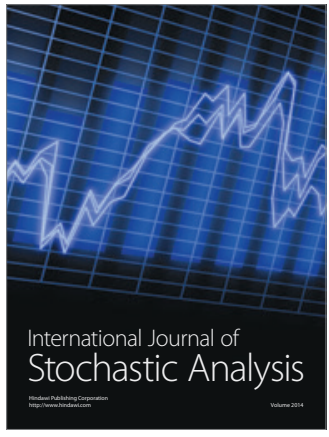

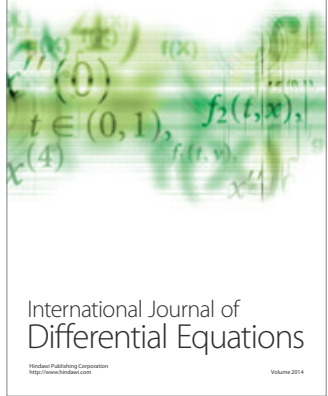
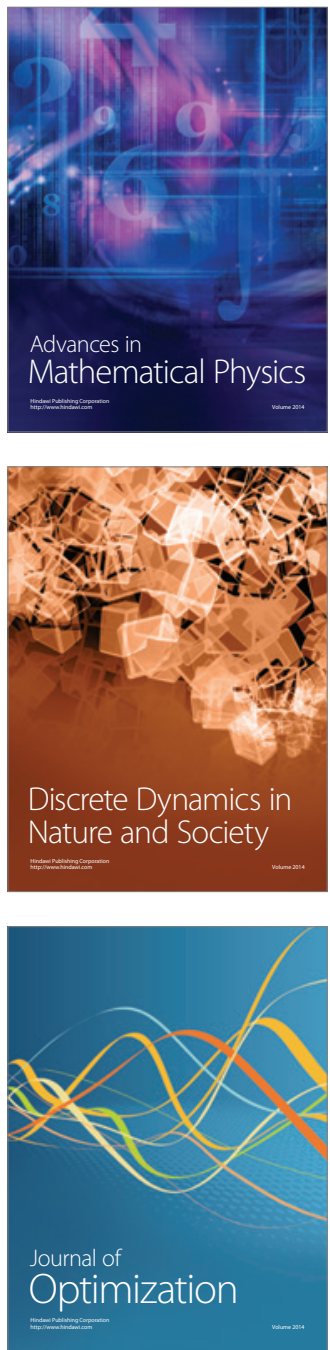